\title{
Diseño de itinerarios de aprendizaje para el desarrollo progresivo de la competencia transversal "Innovación, creatividad y emprendimiento"
}

Andrés Boza ${ }^{\mathrm{a}}$, Llanos Cuenca ${ }^{\mathrm{a}}$, Marta Fernández-Diego ${ }^{\mathrm{a}}$, Leonor Ruiz ${ }^{\mathrm{a}}$, Faustino Alarcón $^{\text {b }}$, Jose Manuel Prats-Montalbán ${ }^{\text {b }}$, M.M.E. Alemany ${ }^{\text {b }}$, Mariluz Gordo ${ }^{\text {a }}$

${ }^{a}$ Escuela Técnica Superior de Ingeniería Informática,${ }^{b}$ Escuela Técnica Superior de Ingeniería Industrial. Universitat Politècnica de València (España) (aboza@omp.upv.es,llcuenca@omp.upv.es,marferdi@omp.upv.es,Iruiz@omp.upv.es, faualva@omp.upv.es,jopramon@eio.upv.es,mareva@omp.upv.es,magormon@upvnet. upv.es)

\begin{abstract}
The level with which our students arrive with respect to a certain transversal competence is often unknown and initially presupposed by the teacher. The proposal for innovation and educational improvement presented in this document focuses on the early identification of this initial level in the transversal competence "Innovation, creativity and entrepreneurship" in order to offer learning activities at different levels to reinforce those aspects required for an adequate coverage of the competence. For the latter, it is proposed designing a map of classified learning activities. The desing of this map enables to have a broad view of the activities carried out for the development of competence, to identify overlaps of activities at the same level, and to identify levels not addressed by no activity, which encourages the development of activities that cover levels not reached by other activities.
\end{abstract}

Keywords: Learning Activities, Transversal Competences, Map of Activities, Teaching-Learning Process, Creativity, Innovation, Entrepreneurship,

\footnotetext{
Resumen

El nivel con el que llegan nuestros estudiantes respecto a una determinada competencia trasversal, es en muchas ocasiones desconocido e inicialmente presupuesto por el profesor. La propuesta de innovación y mejora educativa presentada en este documento se centra en la identificació temprana de ese nivel de partida de los alumnos en la competencia trasversal "Innovación, creatividad y emprendimiento" para poder ofrecerles actividades de aprendizaje a diferentes niveles con el fin de reforzar aquellos aspectos requeridos para una adecuada cobertura de la competencia. Para esto último, se propone el diseño de un mapa de actividades de aprendizaje clasificado que permita tener una visión amplia de las actividades llevadas a cabo para el desarrollo de la competencia, identificar superposiciones de actividades en el mismo nivel, e identificar niveles no abordados por ninguna actividad, lo que fomenta el desarrollo de actividades que cubren niveles no alcanzados por otras actividades.
} 
Palabras clave: Actividades de aprendizaje, Competencias Trasversales, Mapa de Actividades, Proceso Enseñanza-Aprendizaje, Creatividad, Innovación, Emprendimiento

\section{Introducción}

Las universidades estan rediseñando sus planes de estudios de acuerdo a nuevos perfiles profesionales que requiere la sociedad. Para estos nuevos perfiles son necesarias competencias específicas, pero también compentencias transversales, o también denominadas genéricas. (Perrenoud, 2008) define competecias como el conjunto de conocimientos, habilidades y actitudes capaces de movilizar una persona de forma integrada para responder efectivamente a un tipo de situaciones. Las competencias específicas se hubican en una determinada área de conocimiento y afectan a los perfiles profesionales que se pueden desarrollar dentro de esa área. Las competencias transversales son genéricas y transferibles en diferentes contextos personales, sociales, académicos y profesionales a lo largo de la vida (Boza et al., 2017).

De forma general, el nivel de partida de un alumno en relación a competencias no transversales puede ser conocido por la estructura de los planes de estudios previamente cursados. Así, el profesor tiene como referencia un determinado nivel en los alumnos sobre las competencias específicas que debe abordar debido al conocimiento de la formación recibida anteriormente. Sin embargo, cuando se trata de competencias transversales, ese nivel de partida del alumno no es tan fácil de identificar.

Las capacidades del alumno de hablar en público, tener pensamiento crítico o de trabajo en equipo, son, en la mayoría de los casos, desconocidas por el profesor cuando comienza su actividad docente con un grupo nuevo de alumnos. Sin embargo, un importante número del profesorado, continua actuando como con el resto de competencias no transversales, es decir, asumiendo un determinado nivel de partida del alumnado y definiendo actividades de aprendizaje para la competencia según ese "supuesto" nivel de partida.

La experiencia adquirida en anteriores proyectos ha demostrado que una actividad, para el desarrollo de competencias transversales, llevada a cabo en el aula puede tener resultados diferentes en los estudiantes. No todos se enfrentan de igual forma a la actividad, les resulta difícil, o excesivamente fácil, y surgen bloqueos o falta de motivación.

La competencia tranversal "Innovación, Creatividad, y Emprendimiento" no escapa de esta problematica cuando las actividades están más enfocadas a los indicadores de la competencia que a las necesidades del alumno. Esta compentencia esta incluida en el proyecto UPV de competencias transversales puesto en marcha por la Universitat Politècnica de València. Este proyecto incluye 13 competencias genéricas (Universitat Politècnica de Valencia, 2017).

La propuesta presentada en este artículo se centra en primer lugar en identificar el nivel de partida de la competencia de los alumnos para adecuar el proceso enseñanza-aprendizaje a los diferentes niveles encontrados en el aula, y por otro en la identificación y clasificación de actividades de aprendizaje en diferentes niveles dentro de la competencia.

(cc) EY-NC-ND 2018, Universitat Politècnica de València

Congreso IN-RED (2018) 
La propuesta incorpora al alumno como elemento clave en el proceso enseñanzaaprendizaje.

Esta propuesta se enmarca dentro de un proyecto de innovación educativa en desarrollo y lo que se presenta aquí es el diseño de dicha propuesta y el despliege que se esta desarrollando. Un aspecto clave es la intencionalidad de los autores para que la propuesta pueda analizarse desde la perspectiva de otras competencias trasversales. Así, la competencia "innovación, creatividad y emprendimiento" se presenta como soporte de la propuesta, aunque otras competencias trasversales podrían ser abordadas de forma similar. Además, la propuesta no se focaliza en un nivel de grado o master, ni para una determinada titulación al tratarse de una competencia trasversal.

\subsection{Objetivos}

Así, el objetivo general es identificar los diferentes niveles de partida en la competencia “Creatividad, Innovación y Emprendimiento” a cada alumno y clasificar las actividades de aprendizaje disponibles para dicha competencia para luego poder definir diferentes líneas de actuación que favorezcan su adecuado alcance de la competencia.

De forma más concreta, los objetivos a cubrir son:

1. Diseñar la escala para identificar niveles en el alcance de la competencia.

2. Diseñar actividades iniciales de pre-evaluación que permitan identificar la capacidad creativa, de innovación y emprendimiento para cada alumno.

3. Identificar y clasificar actividades de acuerdo con la escala establecida para construir un mapa de actividades.

\section{Diseño de la escala para identificar niveles de alcanze de la competencia}

El diseño de la escala se justifica por el requerimiento de medir el nivel de los alumnos respecto a la competencia, así como, el nivel en el que mejor contribuyen las actividades de aprendizaje al alcance de la competencia. Medir significa asignar números, símbolos o valores a las propiedades de los objetos o eventos de acuerdo con reglas(Stevens, 1951). En ciencias sociales se trabaja con conceptos abstractos que no pueden caracterizarse como objeto o evento. En este ámbito definen medición como el proceso de vincular conceptos abstractos con indicadores empíricos, el cual se realiza mediante un plan explícito y organizado para clasificar (y con frecuencia cuantificar) los datos disponibles (los indicadores), en términos del concepto que el investigador tiene en mente(Carmines \& Zeller, 1979), en nuestro caso para identificar niveles de alcance de la competencia. Para (Grinnell Jr \& Unrau, 2005) la función de la medición es establecer una correspondencia entre el "mundo real” y el "mundo conceptual”. El primero provee evidencia empírica, el segundo proporciona modelos teóricos para encontrar sentido a ese segmento del mundo real que estamos tratando de describir.

(c) ) EY-NC-ND 2018, Universitat Politècnica de València 
En este proceso, el instrumento de medición de datos tiene un papel central. Sin él no hay observaciones clasificadas. Así, los registros del instrumento de medición representan valores visibles de conceptos abstractos. Aunque no hay medición perfecta, debemos acercarnos lo más posible a la representación fiel de las variables que se van a observar mediante el instrumento de medición(Grinnell Jr \& Unrau, 2005).

Existen tres cuestiones básicas respecto a las variables que deben considerarse al momento de construir un instrumento(Hernandez Sampieri, Fernandez Collado, \& Baptista Lucio, 2014): la operacionalización, la codificación y el establecimiento de los niveles de medición.

1) El tecnicismo “operacionalización de variables” se emplea en investigación científica para designar al proceso mediante el cual se transforma la variable de conceptos abstractos a términos concretos, observables y medibles, es decir, dimensiones e indicadores(Arias, 2006). Este proceso incluye las etapas de definición nominal y conceptual de la variable, la dimensionalización de la misma (determinando sus factores, dimensiones o componentes), definir indicadores para las dimensiones y finalmente identificar, desarrollar o generar ítems o valores de cada indicador.

2) La codificación de datos significa asignarles un valor numérico o símbolo que los represente. Es decir, a las categorías (opciones de respuesta) de cada ítem o variable se les asignan valores numéricos o signos que tienen un significado. Por ejemplo, la Categoría Masculino - Femenino se puede codificar como 1 - 2, o el ítem "En esta empresa tengo libertad para tomar decisiones” se puede codificar como 5. Totalmente de acuerdo, 4. De acuerdo, 3. Ni de acuerdo ni en desacuerdo, 2. En desacuerdo, 1. Totalmente en desacuerdo)

3) Los niveles de medición más conocidos son: i) Nivel de medición nominal, donde hay dos o más categorías del ítem o la variable, y estas categorías no tienen orden ni jerarquía. (Ej. La variable “género” de la persona tiene las categorías: masculino y femenino. ii) Nivel de medición ordinal, donde hay varias categorías que mantienen un orden de mayor a menor (Por ej. Presidente es más que vicepresidente, y a su vez, más que director general, pero no se puede precisar cuánto más). iii) Nivel de medición por intervalos. Además del orden o la jerarquía entre categorías, se establecen intervalos iguales en la medición, es decir, la distancias entre categorías son las mismas a lo largo de toda la escala (Por ej. La variable "número de empleados a su cargo").

En el caso de las competencias transversales, el diseño de la escala es complejo y es dependiente del enfoque del profesor o profesores (diseñadores de la escala) quieran dar en el análisis de dicha competencia transversal y de la propia naturaleza de la competencia. Por ejemplo, la competencia transversal “Aprendizaje permanente” tiene unas dimensiones muy diferentes de la competencia “Comunicación Efectiva” tanto por las características intrínsecas de la competencia, como por los aspectos subjetivos del profesor o profesores a la hora de definir las dimensiones y los indicadores.

En el caso de la competencia transversal de la UPV "Innovación, Creatividad y Emprendimiento” la competencia se aborda como un proceso. En concreto, tomando los

(cc) BY-NC-ND 2018, Universitat Politècnica de València

Congreso IN-RED (2018) 
descriptores de las definiciones de innovación, creatividad y emprendimiento se establece un proceso a seguir, que contiene los siguientes pasos(Boza et al., 2017):

- Se analiza el entorno en busca de oportunidades

- Se proponen ideas creativas

- Se llevan a la práctica o se indica cómo hacerlo, emprendimiento, ejecución

- Se analizan riesgos y beneficios, valor de la innovación.

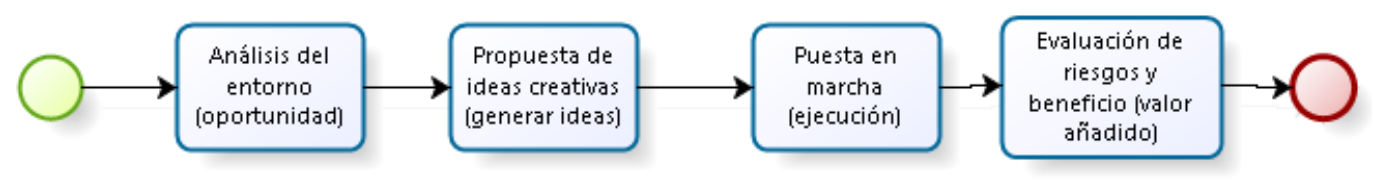

Fig 1: Proceso asociado a la competencia de creatividad, innovación y emprendimiento (Boza et al., 2017).

Así, la operacionalización de la competencia en dimensiones, indicadores e ítems se presenta en la siguiente tabla:

\begin{tabular}{|c|c|c|c|}
\hline Variable & Dimensión & Indicador & Item \\
\hline \multirow{4}{*}{$\begin{array}{l}\text { Competencia Transversal } \\
\text { "Innovación, Creatividad y } \\
\text { Emprendimiento" }\end{array}$} & $\begin{array}{l}\text { Análisis del entorno } \\
\text { (oportunidad }\end{array}$ & $\begin{array}{l}\text { El alumno se cuestiona la } \\
\text { realidad }\end{array}$ & \multirow{4}{*}{$\begin{array}{l}\text { Categorías: } \\
\text { D. No alcanzado } \\
\text { C. En desarrollo } \\
\text { B. Bien/adecuado } \\
\text { A. } \\
\text { Excelente/ejemplar }\end{array}$} \\
\hline & $\begin{array}{l}\text { Propuesta de ideas } \\
\text { creativas (generar ideas) }\end{array}$ & Aporta ideas & \\
\hline & $\begin{array}{l}\text { Puesta en marcha } \\
\text { (ejecución) }\end{array}$ & $\begin{array}{l}\text { Plasma de manera formal } \\
\text { las ideas }\end{array}$ & \\
\hline & $\begin{array}{l}\text { Evaluación de riesgos y } \\
\text { beneficio (valor añadido) }\end{array}$ & Identifica resultados & \\
\hline
\end{tabular}

Tabla 1. Operacionalización de la competencia transversal "Innovación, Creatividad y Emprendimiento".

La tabla anterior debe entenderse como una de las maneras de realizar la operacionalización de la competencia, ya que como se ha indicado anteriormente, los diseñadores de cada escala están condicionados por la naturaleza de cada competencia transversal, y su propia perspectiva. Así, para otras escalas se pueden requerir mediciones de 0 a 10 , utilizar escalas de Likert o usar diferenciales semánticos (por ej. El alumno es muy creativo --- El alumno es poco creativo). 
Respecto a los niveles de medición, para el caso que nos ocupa (identificación del nivel del estudiantes en la competencia trasversal "Innovación, creatividad y emprendimiento) se requieren al menos dos niveles. El enfoque que ha seguido esta propuesta es el establecer los niveles como un camino ordenado para el alcance de la competencia, de forma que los niveles a utilizar son el nivel de medición ordinal o el nivel de medición por intervalos.

La propuesta desarrollada en el presente artículo se enfoca en identificar el nivel inicial de los estudiantes en la competencia y clasificar actividades de aprendizaje de acuerdo a estos niveles para adaptar el proceso de enseñanza aprendizaje a los diferentes niveles encontrados en el aula. Esta es la razón por la que se require la escala ordenada. Sin embargo, para otras propuestas también se puede utilizar el nivel de medición nominal cuando se quiera poner el foco en las dimensiones en sí mismas y no tanto en su evolución (por ej. "La actividad aborda” Categorias: Innovación / Creatividad / Emprendimiento).

\section{Diseño de actividades iniciales de pre-evaluación que permitan identificar la capacidad creativa, de innovación y emprendimiento para cada alumno.}

El diseño de una actividad o actividades para la identificación temprana del nivel inicial del alumno en la competencia trasversal es un reto debido a la complejidad de la competencia al incluir elementos relacionados pero que pueden ser abordados de forma independiente.

Así, se podría diseñar una actividad que aborde de forma global los tres elementos que componen la competencia "Innovación, Creatividad y Emprendimiento", o diseñar actividades separadas para la evaluación de cada uno de estos componentes.

No hay que olvidar que el propósito de la actividad no es que sirva como una herramienta de aprendizaje para el alumno, sino que sirva como herramienta para la medición del nivel inicial de la competencia del alumno.

En la búsqueda de estas actividades nos podemos encontrar propuestas ampliamente utilizadas como the Torrance test of creative thinking (Torrance, 1974) y a su vez, críticas a estas mismas propuestas como (Piffer, 2012) que cuestiona que el test de Torrance aborde creative thinking, ya que pone el foco en el pensamiento divergente dejando de lado aspectos también importantes para la creatividad como el pensamiento convergente. Esta complejidad surge de igual modo si hablamos de innovación o de emprendimiento.

\subsection{Evaluación del nivel inicial del cada alumno}

Las actividades propuestas en el anterior paso persiguen la idenficiación temprana del nivel inicial del estudiante. En este paso se persigue el despliege de dichas actividades en el aula para realizar la evaluación.

Para dicho despliege hay que considerar dos aspectos importantes:

1. Identificación termprana. Desde el punto de vista temporal, la actividad debe realizarse en las primeras sesiones de puesta en marcha de la asignatura. Así, el profesor puede reaccionar y actuar en función de los resultados.

(cc) EY-NC-ND 2018, Universitat Politècnica de València

Congreso IN-RED (2018) 
2. Nivel de los estudiantes. Desde una perspectiva individual o de grupo, la actividad debe realizarse de forma individualizada y poder conocer los resultados de forma individualizada para poder ofrecer propuestas personalizadas a cada alumno.

\subsection{Identificar el nivel de cada estudiante en la competencia trasversal.}

Como resultado de la anterior evaluación, podemos identificar el nivel de cada estudiante en la competencia trasversal. Esto permite la agrupación de los alumnos por niveles para poder actuar de forma particular dependiendo del nivel en la competencia.

Un ejemplo puede verse con más detalle en la siguiente gráfica donde se trabaja con un grupo de 50 alumnos y se utiliza una escala de 0 a 10 respecto al nivel inicial del alumno en la competencia. Cada punto representa a un alumno:

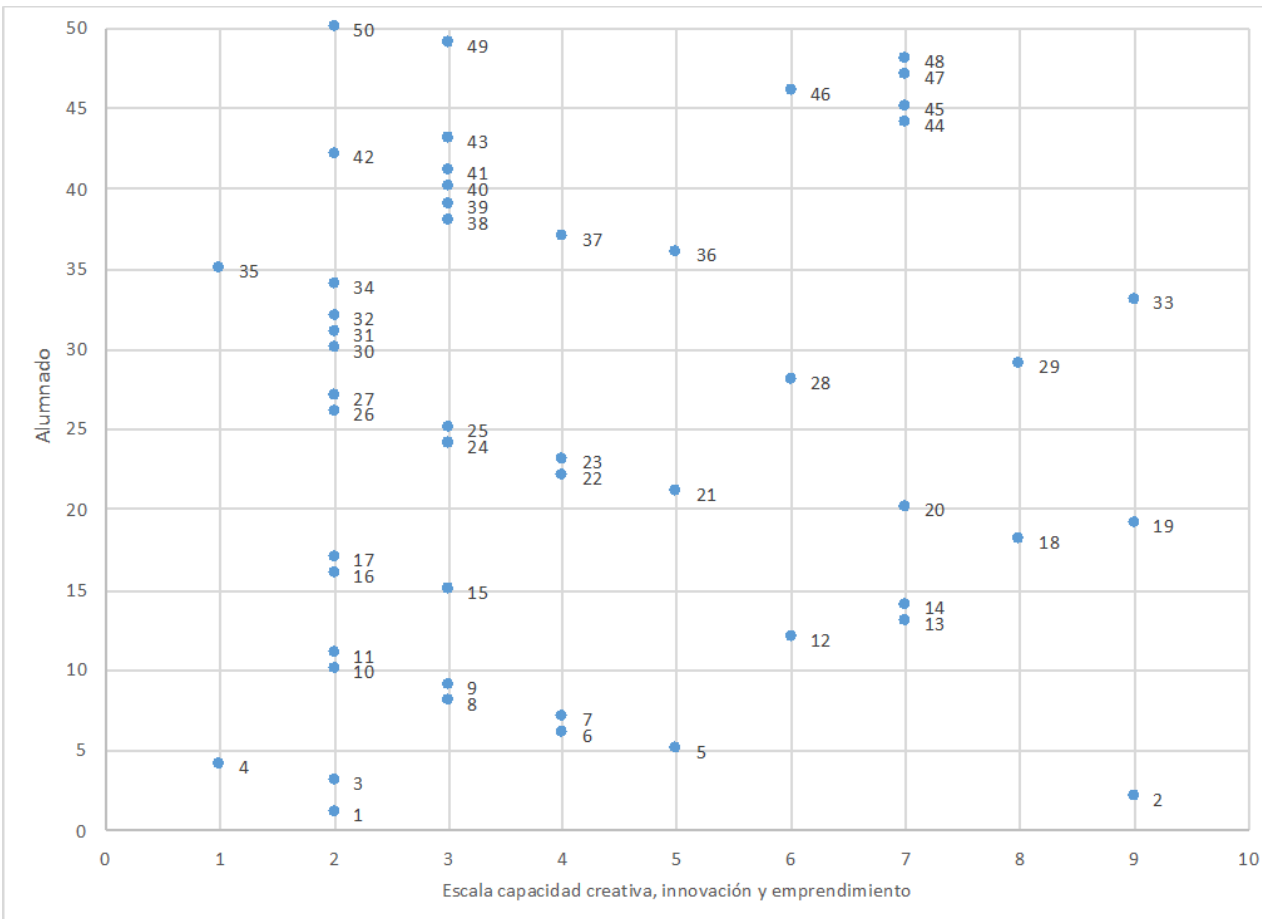

Fig 2. Nivel de la competencia para cada alumno.

La gráfica representa el diagrama de dispersión de los resultados obtenidos. Para dicho ejemplo, la capacidad creativa, de innovación y emprendimiento de los alumnos 33, 19 y 2 difiere bastante de la de los alumnos 35 y 4, por lo que las actividades para el desarrollo de la competencia a realizar en estos dos grupos de alumnos deberían ser diferentes. Así, se deberían establecer grupos de alumnos para el desarrollo de las actividades en función de su capacidad y potencial inicial. 


\section{Identificar y clasificar actividades de acuerdo con la escala establecida para construir un mapa de actividades.}

La construcción del mapa requiere en primer lugar identificar actividades de aprendizaje orientadas a la adquisición de la competencia transversal. El trabajo previamente realizado de operacionalización donde se identifican dimensiones de la competencia facilita esta labor, ya que las actividades deberían abarcar una o más de estas dimensiones. Este trabajo es más sencillo cuando ya se están realizando actividades de aprendizaje en la asignatura y ya tenemos un conjunto de actividades de aprendizaje identificadas. Si todavía no se ha trabajado con la competencia transversal, se deben buscar e identificar actividades de aprendizaje de la misma, con lo que este proceso será algo más largo.

En segundo lugar, se deben clasificar las actividades de acuerdo con la escala establecida para construir el mapa de actividades. Es decir, identificar el nivel (o niveles) para cada actividad de aprendizaje. Con ello, es posible construir el mapa de actividades situando todas las actividades en el mapa de acuerdo con la escala definida. Así, es posible identificar las actividades más idóneas para cada nivel de la escala.

En la figura 3 se muestra un ejemplo del mapa utilizando una escala de alcance de la competencia de 0 a 10 . El mapa nos muestra el conjunto de actividades de aprendizaje diseñadas para la competencia así como su ubicación dentro de la escala.

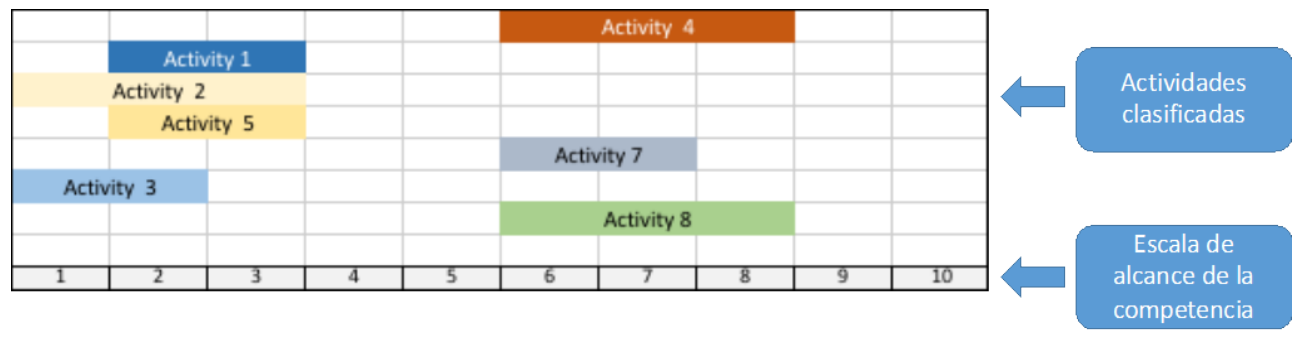

Fig 3. Mapa de actividades de apredizaje clasificadas por nivel.

La ubicación de las actividades en el mapa permite: a) por un lado identificar conjuntos de actividades que están trabando el mismo nivel, por lo que quizás no es necesario desplegar en el aula todo este conjunto de actividades, ya que con la realización de una de dichas actividades ya se tendría cubierto dicho nivel (por ejemplo el nivel 2 está saturado de actividades ya que se trabaja con las actividades 1, 2, 3 y 5). Y por otro lado, b) identificar niveles que no son trabajados por ninguna actividad. En la figura 4 se muestra estos GAPs.

(cc) EY-NC-ND 2018, Universitat Politècnica de València

Congreso IN-RED (2018) 


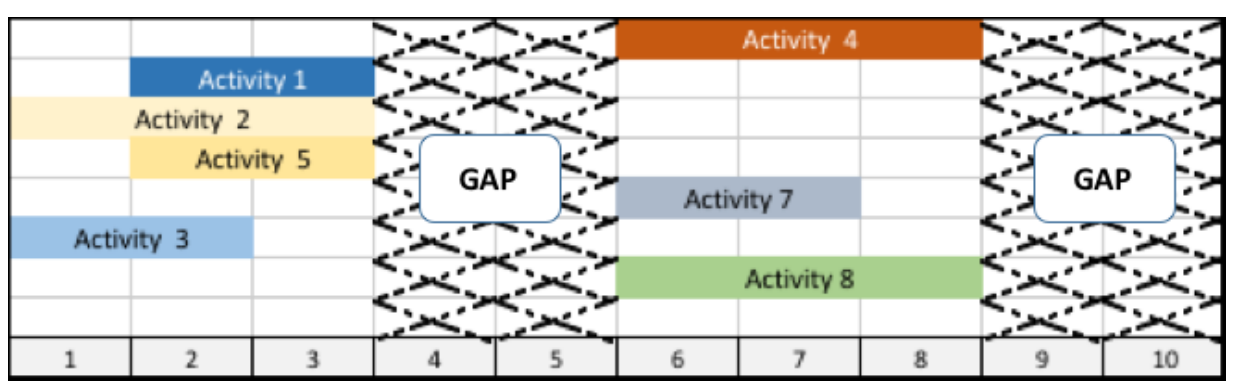

Fig 4. GAPs en el mapa de actividades de apredizaje clasificadas por nivel.

La identificación de estos GAPs permite pasar a la siguiente etapa en la configuración del mapa de actividades.

\subsection{Completar el mapa de actividades para cubrir rangos no cubiertos mediante el diseño de nuevas actividades}

La búsqueda de nuevas actividades para trabajar la competencia transversal se facilita con la construcción del mapa siguiendo las etapas anteriores, ya que sirve como guía para la búsqueda de actividades no cubiertas. Es decir, el proceso de búsqueda y análisis de actividades de aprendizaje queda dirigido por el objetivo de cubrir los GAPs encontrados. La figura 4 muestra esta inclusión de dos nuevas actividades (la 9 y la 10) para cubrir estos GAPs.

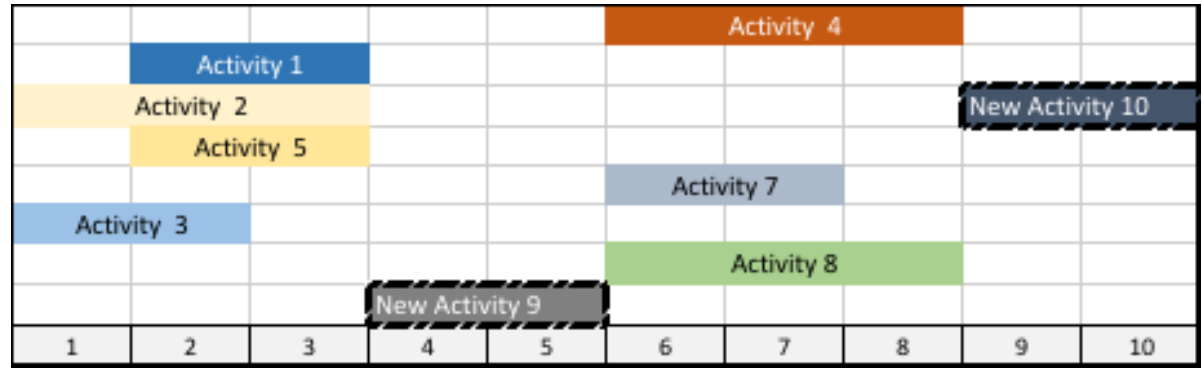

Figure 5: Incorporación de actividades para cubrir los GAPS

\section{Conclusiones}

El problema de acercarse a las necesidades particulares de cada alumno a la hora de enfrentarse y desarrollar la competencia "innovación, creatividad y emprendimiento" se ha abordado mediante la identificación temprana del nivel del estudiante en la competencia y el diseño de un mapa de actividades.

La línea seguida en este paper no ha pretendido focalizarse en una única competencia transversal, sino que se ha intentado abordado desde una perspectiva general para ser transferible a diferentes competencias transversales. Asi, como se ha indicado anteriormente, para las diferentes competencias transversales será necesario adecuarse a la propia naturaleza de la competencia, y las escalas de medición pueden estar condicionadas por el enfoque del diseñador de la misma respecto a la competencia.

(c) EY-NC-ND 2018, Universitat Politècnica de València 
Las etapas abordadas para dicho propósito con (i) el diseño de la escala para identificar niveles de alcanze de la competenciam, y (ii) el diseño de actividades iniciales de preevaluación que permitan identificar la capacidad creativa, de innovación y emprendimiento para cada alumno permiten adaptar el proceso de enseñanza aprendizaje a los diferentes niveles encontrados en el aula. Así, para adaptar dicho proceso, (iii) la identificación y clasificación de actividades de acuerdo con la escala de establecida permiten la construcción de un mapa de actividades acordes para cada nivel.

La identificación temprana del nivel del estudiante en la competencia trasvesal permite:

1. Tener una visión amplia de los estudiantes en nuestro aula sobre su punto de partida en la competencia.

2. Identificar clusters de estudiantes del mismo nivel respecto a la competencia

3. Adaptar el proceso de enseñanza-aprendizaje de acuerdo con estos grupos.

Además, el diseño del mapa de actividades de aprendizaje clasificadas permite:

1. Tener una visión amplia de las actividades llevadas a cabo para el desarrollo de la competencia.

2. Identificar superposiciones de actividades en el mismo nivel, lo que plantea la cuestión de si eliminar o no alguna de las actividades.

3. Identificar niveles no abordados por ninguna actividad, lo que fomenta el desarrollo de actividades que cubren niveles no alcanzados por otras actividades.

4. Ofrecer a los estudiantes el mapa de actividades como una herramienta para su entrenamiento.

Esta propuesta incorpora al estudiante como un elemento clave en el proceso de enseñanzaaprendizaje. Y, el mapa de actividades se convierte en una herramienta para los estudiantes, donde pueden identificar las actividades por nivel y trabajar en mayor medida en las más adecuadas de acuerdo a sus necesidades y potencial.

\section{Agradecimientos}

Esta investigación se ha llevado a cabo en el marco del Proyecto de Innovación y Mejora Educativa (PIME 2017-18 Ref. A10) de la Universitat Politècnica de València.

\section{Referencias}

Arias, F. (2006). El proyecto de investigación. Introducción a La Metodología Científica. http://doi.org/Q180.55-M4A7

Boza, A., Fernández-Diego, M., Ruiz, L., Gordo, M., Alemany, M. M. E., Alarcón, F., \& Cuenca, L. (2017). Transversal Competences as a Medium of Teaching. The Case of

(cc) EY-NC-ND 2018, Universitat Politècnica de València

Congreso IN-RED (2018) 
Creativity, Innovation and Entrepreneurship. In Strategic Innovative Marketing (pp. 3-10). Springer.

Carmines, E. G., \& Zeller, R. A. (1979). Reliability and validity assessment. Quantitative Applications in the Social Sciences. http://doi.org/10.1037/018269

Grinnell Jr, R. M., \& Unrau, Y. (2005). Social work research and evaluation: Quantitative and qualitative approaches. Cengage Learning.

Hernandez Sampieri, R., Fernandez Collado, C., \& Baptista Lucio, M. del P. (2014). Metodología de la investigación. Metodología de la investigación. Retrieved from http://www.casadellibro.com/libro-metodologia-de-la-investigacion-5-ed-incluye-cdrom/9786071502919/1960006

Perrenoud, P. (2008). Diez nuevas competencias para enseñar. Tiempo de Educar, 9(17), 159.

Piffer, D. (2012). Can creativity be measured? An attempt to clarify the notion of creativity and general directions for future research. Thinking Skills and Creativity, 7(3), 258264. http://doi.org/10.1016/j.tsc.2012.04.009

Stevens, S. S. (1951). Mathematics, measurement, and psychophysics. In Handbook of Experimental Psychology (pp. 1-49).

Torrance, E. (1974). The Torrance test of creative thinking: Norms-technical manual. Bensenville, Illinois: Scholastic Testing Services. Inc.

Universitat Politècnica de Valencia. (2017). Conoce el Proyecto de Competencias Transversales UPV. http://www.upv.es/contenidos/COMPTRAN/. Retrieved from http://www.upv.es/contenidos/COMPTRAN/ 